\title{
Efficient Dispersion of Mobile Agents without Global Knowledge *
}

\author{
Takahiro Shintaku ${ }^{1}$, Yuichi Sudo ${ }^{\dagger 1}$, Hirotsugu Kakugawa ${ }^{2}$, and Toshimitsu Masuzawa ${ }^{1}$ \\ ${ }^{1}$ Osaka University, Japan \\ ${ }^{2}$ Ryukoku University, Japan
}

\begin{abstract}
We consider the dispersion problem for mobile agents. Initially, $k$ agents are located at arbitrary nodes in an undirected graph. Agents can migrate from node to node via an edge in the graph synchronously. Our goal is to let the $k$ agents be located at different $k$ nodes with minimizing the number of steps before dispersion is completed and the working memory space used by the agents. Kshemkalyani and Ali [ICDCN, 2019] present a fast and space-efficient dispersion algorithm with the assumption that each agent has global knowledge such as the number of edges and the maximum degree of a graph. In this paper, we present a dispersion algorithm that does not require such global knowledge but keeps the asymptotically same running time and slightly smaller memory space.
\end{abstract}

\section{Introduction}

We consider the dispersion problem of mobile robots, which we call mobile agents (or just agents) in this paper. At the beginning of an execution, $k$ agents are arbitrarily placed in an undirected graph at the beginning of an execution. The goal of this problem is to let all agents be located at different nodes. This problem was originally formulated by Augustine and Moses Jr. [1] in 2018. The most interesting point of this problem is the uniqueness of the computation model. Unlike many problems of mobile agents on graphs, we cannot access the identifiers of the nodes and cannot use a local memory at each node, usually called whiteboard. In this setting, an agent cannot get/store any information from/on a node when it visits the node. Instead, $k$ agents have unique identifiers and can communicate with each other when they visit the same node in a graph. The agents must coordinately solve a common task via direct communication with each other.

Several algorithms were presented for the dispersion problem of mobile agents in the literature. Augustine and Moses Jr. 1 gave an algorithm that achieves dispersion within $O\left(m^{\prime}\right)$ steps, where $m$ and $\delta_{\max }$ are the number of edges and the maximum degree of a graph, respectively, and $m^{\prime}=\min \left(m, k \delta_{\max } / 2,\left(\begin{array}{c}k \\ 2\end{array}\right)\right)$. In this algorithm, each agent uses $O\left(k \log \left(\delta_{\max }+k\right)\right)$ bits of memory space. Kshemkalyani and Ali [3] presents three dispersion algorithms. The first one slightly decreases the memory space per agent from $O\left(k \log \left(\delta_{\max }+k\right)\right)$ bits to $O\left(\ell \log \left(\delta_{\max }+k\right)\right)$ bits, where $\ell$ is the number of nodes at which at least one agent is located at the beginning of an execution. The second one uses $O\left(d \log \delta_{\max }\right)$ bits per agent and solves the dispersion problem within $O\left(\delta_{\max }^{d}\right)$ steps, where $d$ is the diameter of a graph. The third one achieves dispersion with much smaller space: $O\left(\log \left(\delta_{\max }+k\right)\right)$ bits per agent. However, it requires $O\left(m^{\prime} \ell\right)$ time steps before dispersion is achieved. The current state of art algorithm was given by Kshemkalyani, Molla, and Sharma [4. This algorithm is both time and space efficient. The running time is $O\left(m^{\prime} \log \ell\right)$

\footnotetext{
*This work was supported by JSPS KAKENHI Grant Numbers 19H04085, 19K11826, and 20H04140 and JST SICORP Grant Number JPMJSC1606.

${ }^{\dagger}$ Corresponding author:y-sudou[at]ist.osaka-u.ac.jp
} 
Table 1: Dispersion Algorithm for Arbitrary Undirected Graphs. $\left(m^{\prime}=\min \left(m, k \delta_{\max } / 2,\left(\begin{array}{l}k \\ 2\end{array}\right)\right)\right.$

\begin{tabular}{cccc}
\hline & Memory Space & Running Time & Knowledge \\
\hline$[1]$ & $O\left(k \log \left(\delta_{\max }+k\right)\right)$ & $O\left(m^{\prime}\right)$ & \\
{$[3]$} & $O\left(\ell \log \left(\delta_{\max }+k\right)\right)$ bits & $O\left(m^{\prime}\right)$ steps & \\
{$[3]$} & $O\left(d \log \delta_{\max }\right)$ bits & $O\left(\delta_{\max }^{d}\right)$ steps & \\
{$[3]$} & $O\left(\log \left(\delta_{\max }+k\right)\right)$ bits & $O\left(m^{\prime} \ell\right)$ steps & \\
{$[4]$} & $O(\log n)$ bits & $O\left(m^{\prime} \log \ell\right)$ steps & $m, k, \delta_{\max }$ \\
this work & $O\left(\log \left(\delta_{\max }+k\right)\right)$ bits & $O\left(m^{\prime} \log \ell\right)$ steps & \\
\hline
\end{tabular}

steps and the memory space used by each agent is $O(\log n)$ bits, where $n$ is the number of nodes in a graph. However, this algorithm requires global knowledge, i.e., $m, k$, and $\delta_{\max }$, as mentioned in their paper. 4]. To the best of our understanding, their algorithm does not necessarily require the exact values of those parameters. It requires only upper bounds $M, K$, and $\Delta$ on $m, k$, and $\delta_{\max }$, respectively. Then, the assumption becomes much weaker, but time and space complexities may increase depending on how large those upper bounds are. Indeed, given those upper bounds, the algorithm achieves dispersion within $O\left(M^{\prime} \log \ell\right)$ steps and uses $O\left(\log M^{\prime}\right)$ bits of memory space, where $M=\min \left(M, K \Delta, K^{2}\right)$.

\subsection{Our Contribution}

The main contribution of this paper is removing the requirement of global knowledge of the algorithm given by [4. Specifically, we gave a dispersion algorithm whose running time is $O\left(m^{\prime} \log \ell\right)$ steps and uses $O\left(\log \left(\delta_{\max }+k\right)\right)$ bits of the memory space of each agent. The proposed algorithm does not require any global knowledge such as $m, k$, and $\delta_{\max }$. In addition, the space complexity is slightly smaller than the algorithm given by [4, while the running times of both algorithms are asymptotically the same.

As with the existing algorithms listed in Table1, the proposed algorithm works on an arbitrary simple, connected, and undirected graph. This algorithm solves the dispersion problem regardless of the initial locations of the agent in a graph. We require that the agents are synchronous, as in the algorithm given by [4].

\subsection{Other Related Work}

The dispersion problem has been studied not only for arbitrary undirected graphs, but for graphs of restricted topology. Augustine and Moses Jr. [1] addressed this problem for paths, rings, and trees. Kshemkalyani, Molla, and Sharma [4] studied the dispersion problem also in grid networks. Very recently, the same authors introduced the global communication model [5]. Unlike the above setting, all agents can always communicate each other regardless of their current locations. They studied the dispersion problem for arbitrary graphs and trees under this communication model.

The exploration problem of mobile agents is closely related to the dispersion problem. This problem requires that each node (or each edge) of a graph is visited at least once by an agent. If the unique node-identifiers are available, a single agent can easily visit all nodes within $2 m$ steps in a simple depth first search traversal. Panaite and Pelc [6] gave a faster algorithm, whose cover time is $m+3 n$ steps. Their algorithm uses $O(m \log n)$ bits in the agent-memory, while it does not use whiteboards, i.e., local memories of the nodes. Sudo, Baba, Nakamura, Ooshita, Kakugawa, and Masuzawa 8 gave another implementation of this algorithm: they removed the assumption of the unique identifiers and reduced the space complexity on the agent-memory from $O(m \log n)$ bits to $O(n)$ bits by using $O(n)$ bits in each whiteboard. The algorithm given by Priezzhev, Dhar, Dhar, and Krishnamurthy [7, which is well known as the rotor-router, also solves the exploration problem efficiently. The agent uses $O\left(\log \delta_{v}\right)$ bits in the whiteboard of each node $v \in V$ and the agent itself is oblivious, i.e., it does not use its memory space at all. The rotor-router algorithm is self-stabilizing, i.e., it guarantees that starting from any (possibly corrupted) configuration, the agent visits all nodes within $O(m D)$ steps [9]. 


\subsection{Organization}

In Section 2, we define the model of computation and the problem specification. In Section 3 , we briefly explain the existing techniques used for the dispersion problem in the literature. This section may help the readers to clarify what difficulties we addresses and how novel techniques we introduce to design the proposed protocol in this paper. In Section 4 we present the proposed protocol. In section [5, we conclude this paper with short discussion for an open problem.

\section{Preliminaries}

Let $G=(V, E)$ be any simple, undirected, and connected graph. Define $n=|V|$ and $m=|E|$. We define the degree of a node $v$ as $\delta_{v}=|\{u \in V \mid(u, v) \in E\}|$. Define $\delta_{\max }=\max _{v \in V} \delta_{v}$, i.e., $\delta_{\max }$ is the maximum degree of $G$. The nodes are anonymous, i.e., they do not have unique identifiers. However, the edges incident to a node $v$ are locally labeled at $v$ so that a robot located at $v$ can distinguish those edges. Specifically, those edges have distinct labels $0,1, \ldots, \delta_{v}-1$ at node $v$. We call this local labels port numbers. We denote the port number assigned at $v$ for edge $\{v, u\}$ by $p_{v}(u)$. Each edge $\{v, u\}$ has two endpoints, thus has labels $p_{u}(v)$ and $p_{v}(u)$. Note that these two labels are not necessarily the same, i.e., $p_{u}(v) \neq p_{v}(u)$ may hold. We say that an agent moves via port $p$ from a node $v$ when the agent moves from $v$ to the node $u$ such that $p_{v}(u)=p$.

We consider that $k$ agents exist in graph $G$, where $k \leq n$. The set of all agents is denoted by $R$. Each agent is always located at some node in $G$, i.e., the move of an agent is atomic and an agent is never located at an edge at any time step (or just step). The agents have unique identifiers, i.e., each agent $a$ has a positive identifier $a$.ID such that $a$.ID $\neq b$.ID for any $b \in R \backslash\{a\}$. The agents knows a common upper bound $i d_{\max }=O(\operatorname{poly}(k))$ such that $i d_{\max } \geq \max _{a \in R} a$.ID, thus the agents can store the identifier of any agent on $O(\log k)$ space. Each agent has a read-only variable $a$.pin $\in\left\{-1,0,1, \ldots, \delta_{v}-1\right\}$. At time step 0 , a.pin $=-1$ holds. For any $t \geq 1$, If $a$ moves from $u$ to $v$ at step $t-1$, a.pin is set to $p_{v}(u)$ at the beginning of step $t$. If $a$ does not move at step $t-1$, a.pin is set to -1 .

The agents are synchronous. All $k$ agents are given a common algorithm $\mathcal{A}$. Let $R(v, t) \subseteq R$ be the set of the agents located at a node $v$. We define $\ell=|\{v \in V \mid R(v, 0) \geq 1\}|$, i.e., $\ell$ is the number of nodes with at least one agent in step 0 . At each step $t \geq 0$, the agents in $R(v, t)$ first communicate with each other and agrees how each agent $a \in R(v, t)$ updates the variables in its memory space in step $t$, including a variable $a$.pout $\in\left\{-1,0,1, \ldots, \delta_{v}-1\right\}$, according to algorithm $\mathcal{A}$. The agents next update the variables according to the agreement. Finally, each agent $a \in R(v, t)$ with $a$.pout $\neq-1$ moves via port $a$.pout. If $a$.pout $=-1$, agent $a$ does not move and stays in $v$ in step $t$.

A node does not have any local memory accessible by the agents. Thus, the agents have to coordinate only by communicating each other. No agents are given a priori any global knowledge such as $m, \delta_{\max }$, and $k$.

The values of all variables in agent $a$ constitute the state of $a$. Let $\mathcal{M}_{\mathcal{A}}$ be the set of all possible agent-states for algorithm $\mathcal{A}$. ( $\mathcal{M}$ may be an infinite set.) Algorithm must specify the initial state $s_{\text {init }}$, which is common to all agents in $R$. A global state of the network or a configuration is defined as a function $C: R \rightarrow(\mathcal{M}, V)$ that specifies the state and the location of each agent $a \in R$. In this paper, we consider only deterministic algorithms. Thus, if the network is in a configuration $C$ at a step, a configuration $C^{\prime}$ in the next step is uniquely determined. We denote this configuration $C^{\prime}$ by next ${ }_{\mathcal{A}}(C)$. The execution $\Xi_{\mathcal{A}}\left(C_{0}\right)$ of $\mathcal{A}$ starting from a configuration $C_{0}$ is defined as an infinite sequence $C_{0}, C_{1}, \ldots$ of configurations such that $C_{t+1}=\operatorname{next}_{\mathcal{A}}\left(C_{t}\right)$ for all $t=0,1, \ldots$

Definition 1 (Dispersion Problem). A configuration $C$ of an algorithm $\mathcal{A}$ is called legitimate if (i) all agents in $R$ are located in different nodes in $C$, and (ii) no agent changes its location in execution $\Xi_{\mathcal{A}}(C)$. We say that $\mathcal{A}$ solves the dispersion problem if execution $\Xi_{\mathcal{A}}\left(C_{0}\right)$ reaches a legitimate configuration for any configuration $C_{0}$ where all agents are in state $s_{\text {init }}$.

We evaluate the running time of algorithm $\mathcal{A}$ as the maximum number of steps until $\Xi_{\mathcal{A}}\left(C_{0}\right)$ reaches 


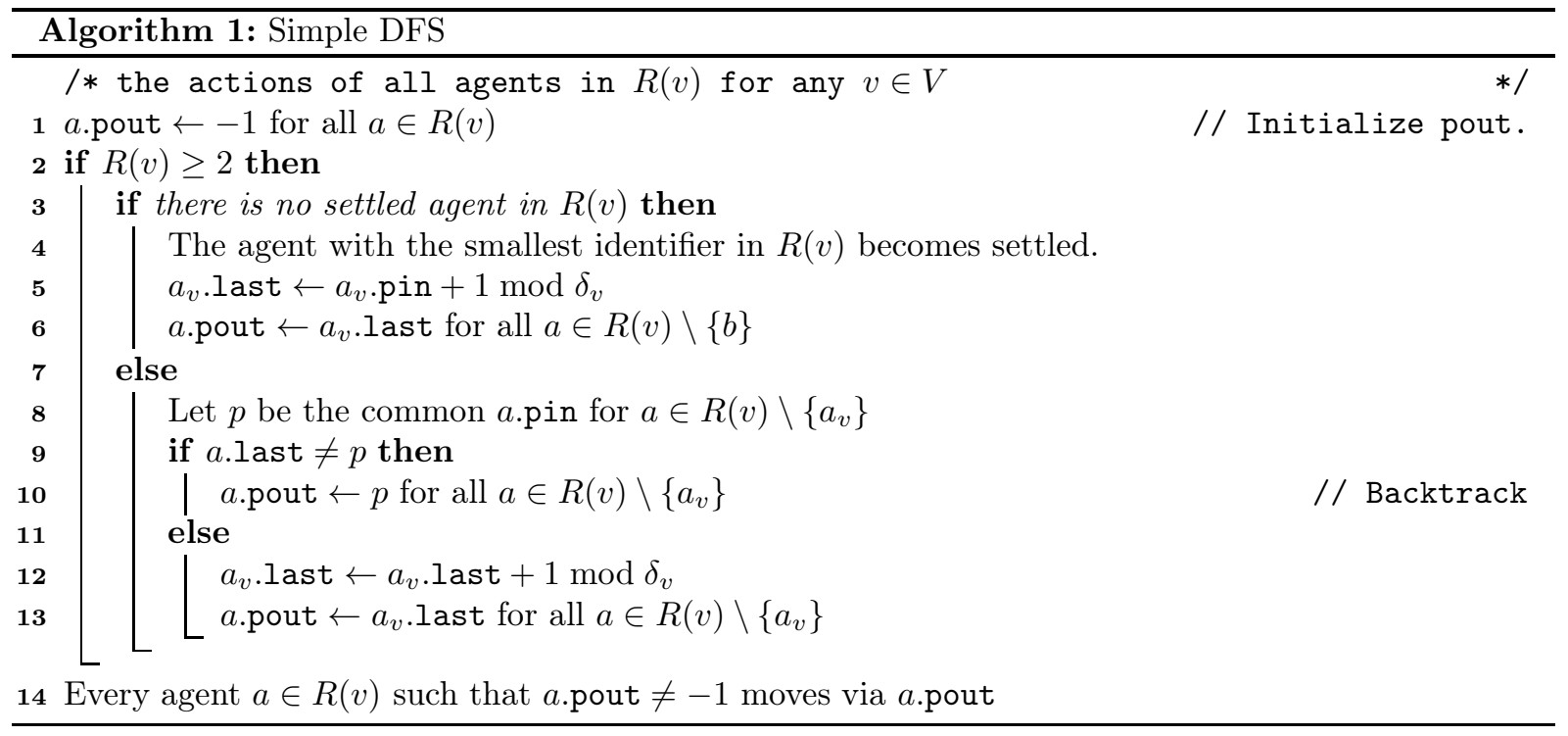

a legitimate configuration, where the maximum is taken over $C_{0}$, any configuration in which all agents are in state $s_{\text {init }}$.

We define $m^{\prime}=\min \left(m, k \delta_{\max } / 2,\left(\begin{array}{c}k \\ 2\end{array}\right)\right)$, which is frequently used in this paper. We simply write $R(v)$ for $R(v, t)$ when time step $t$ is clear from the context.

\section{Existing Techniques}

\subsection{Simple DFS}

If we assume that all $k$ agents are initially located at the same node $v_{\mathrm{st}} \in V$, the dispersion problem can be solved by a simple depth-first search (DFS). The pseudocode of the simple DFS is shown in Algorithm 1. Each agent $a \in R$ maintains a variable mode $\in\{$ settled, unsettled $\}$. We say that agent $a$ is settled if $a$.mode $=$ settled, and unsettled otherwise. All agents are initially unsettled. Once an agent becomes settled at a node, it never becomes unsettled nor moves to another node. We say that a node $v$ is settled if an settled agent is located at $v$; otherwise, $v$ is unsettled. We denote by $a_{v}$ a settled agent located at $v$ when $v$ is settled. (No two agents become settled at the same node.) In this model, no node provides a local memory accessible by agents. However, when a node $v$ is settled, unsettled agents can use the memory of $a_{v}$ like the local memory of $v$ since the unsettled agents and $a_{v}$ can communicate with each other at $v$.

Unsettled agents always move together in a DFS fashion. Each time they find an unsettled node $v$, the agent with the smallest identifier becomes settled at $v$ (Line 4). An settled agent $a_{v}$ maintains a variable $a_{v}$.last $\in\left\{0,1, \ldots, \delta_{v}-1\right\}$ to remember which port was used for the last time by unsettled agents to leave $v$. At step 0 , all agents are unsettled and located at node $v_{\mathrm{st}}$. First, after one agent becomes settled at $v_{\text {st }}$, the other $k-1$ agents move via port 0 . Thereafter, the unsettled agents basically migrates between nodes by the following simple rule: each time they move from $u$ to $v$, it moves via port $p_{v}(u)+1 \bmod \delta_{v}$ (Lines $5,6,12$, and 13). Only exception is the case that node $v$ has already been settled when they move from $u$ to $v$ and $p_{v}(u) \neq a_{v}$.last. At this time, the unsettled agents immediately backtracks from $v$ to $u$ and this backtracking does not update $a_{v}$.last (Line 10).

If $k>n$, this well-known DFS traversal guarantees that the unsettled agents visit all nodes within $4 m$ steps, during which the agents move through each edge at most four times. Since we assume $k \leq n$, the unsettled agents move through at most $m^{\prime}=\min \left(m, k \delta_{\max } / 2,\left(\begin{array}{c}k \\ 2\end{array}\right)\right)$ different edges. Thus, all agents 
become settled 1 within $4 m^{\prime}=O\left(m^{\prime}\right)$ steps, at which point the dispersion is achieved. The space complexity is $O\left(\log \delta_{\max }\right)$ bits per agent: each agent maintains only one non-constant variable last, which requires $\left\lceil\log \delta_{\max }\right\rceil$ bits.

\subsection{Parallel DFS}

Kshemkalyani and Ali 3] generalized the above simple DFS to handle the case that the agents may be initially located at multiple nodes, using $O(\ell \log (\delta+k))$ bits per agent. (Remember that $\ell$ is the number of nodes at which one or more agents are located in step 0 .) That is, $\ell$ groups of unsettled agents perform DFS in parallel. Specifically, in step 0, the agents located at each node $v$ compute $\max \{a$. ID $\mid a \in A(v, 0)\}$ and store it in variable group. Thereafter, the agents use this value as group identifier. Settled agents can distinguish each group by group identifiers, thus they can maintain $\ell$ slots of individual memory space such that unsettled agents of each group can dominantly access one slot of the space. Since simple DFS requires $O\left(\log \delta_{\max }\right)$ bits, this implementation of parallel DFS requires $\left.O\left(\ell \log \delta_{\max }+\ell \log k\right)\right)=O\left(\ell \log \left(\delta_{\max }+k\right)\right)$ bits. The running time is still $O\left(m^{\prime}\right)$ steps.

\subsection{Zombie Algorithm}

We can solve the dispersion with memory space of $O\left(\log \delta_{\max }\right)$ bits per agent at the cost of increasing the running time to $O\left(m^{\prime} k\right)$, regardless the initial locations of $k$ agents. In step 0 , all agents compute its group identifier in the same way as in Section 3.2. However, due to the memory constraints, each settled agent cannot maintain one slot of memory space for each of $\ell$ groups. Instead, it provides only one group with memory space of $O\left(\log \delta_{\max }\right)$ bits. Each settled agent memorizes the largest group identifier it observes: each time unsettled agent visits a node $v, a_{v}$.group is updated to $\max \{a$.group $\mid a \in R(v)\}$. Thus, at least one group of agents can perform its DFS by using the memory space of settled agents exclusively. If an unsettle agent $a$ visits a node $v$ such that $a$.group $<a_{v}$.group, $a$ becomes a zombie, which always chase the agent whose identifier is equal to $a_{v}$.group, which we call the leader of the group. Specifically, a zombie $z$ chases a leader by moving via port $a_{u}$.last each time $z$ visits any node $u$. When $z$ catch up with the leader, $z$ always follow the leader thereafter until $z$ becomes settled, which occurs when $z$ reaches an unsettled node $v$ and $z$ has the smallest identifier among $A(v)$. Every agent moves at most $4 m^{\prime}$ times until it observes larger group identifier than any identifier it has observed so far. Thus, the running time of this algorithm is $O\left(m^{\prime} \ell\right)$ steps.

We call this algorithm the zombie algorithm. The zombie algorithm is essentially the same to the tree-switching algorithm given by [3], while the zombie algorithm is faster only by a constant factor.

\subsection{Dispersion with $O\left(m^{\prime} \log \ell\right)$ steps and $O(\log n)$ bits per agent}

Kshemkalyani, Molla, and Sharma [4] gave the current state of art algorithm that achieves dispersion on arbitrary graphs regardless of the initial locations of the $k$ agents. Their algorithm requires that each agent knows a priori upper bounds $M, K$, and $\Delta$ on $m, k$, and $\delta_{\max }$, respectively. Then, the running time is $O\left(\min \left(M, K \Delta, K^{2}\right) \log \ell\right)$ steps and the required memory space per agent is $O(\log M)$ bits. In particular, if those upper bounds are asymptotically tight, i.e., $N=O(n), M=O(m), K=O(k)$, and $\Delta=O\left(\delta_{\max }\right)$, those complexities are $O\left(m^{\prime} \log \ell\right)$ steps and $O(\log n)$ bits, respectively. In this section, we briefly explain the key idea of their algorithm to clarify how this algorithm requires global knowledge $M, K$, and $\Delta$. The implementation in the following explanation is slightly different from the original implementation in [4, but the difference is not essential.

Let $T$ be a sufficiently large $O\left(\min \left(M, K \Delta, K^{2}\right)\right)$ value. Each unsettled agent maintains a timer variable and counts how many steps have passed since an execution began modulo $T$. Agents switches from stage 1 to stage 2 and from stage 2 to stage 1 in every $T$ steps. At the switch from stage 1 to stage

\footnotetext{
${ }^{1}$ Strictly speaking, according to Algorithm 1, the last one agent never becomes settled even if it visits an unsettled node. However, this does not matter because thereafter the last agent never moves nor change its state.
} 
2 and from stage 2 to stage 1 , all settled agents reset their group identifiers to -1 , which is smaller than any group identifier of unsettled agents.

In stage 1, all unsettled agents at each node $v$ first compare their identifiers and adopts the largest one as their group identifier. Then, unsettled agents perform DFS in parallel like the zombie algorithm. The difference arises when an unsettled agent finds a settled agent with a larger group identifier. Then, instead of becoming a zombie, it stops until the end of stage 1. Moreover, unlike the zombie algorithm, an unsettled agent located at a node $v$ becomes settled even when $|R(v)|=1$.

At the beginning of stage 2 , one or more unsettled agents may be located at settled nodes. Let $G^{\prime}$ be the subgraph induced by all settled nodes in the beginning of stage 2 and $G^{\prime}(v)$ the component that includes a node $v$ in $G^{\prime}$. The goal of stage 2 is to collect all agents in $G^{\prime}(v)$ and locate them to the same node in $G^{\prime}(v)$ for each $v \in V$. Specifically, each unsettled agents located at any node $u$ tries to perform DFS in $G^{\prime}(u)$ twice. Since each settled agent $b$ has only $O(\log M)$ bits space, the memory space of $b$ can be used only by unsettled agents in the group with the largest identifier that $b$ has observed in stage 2 . An settled agents with smaller group identifier stops when it observes a larger group identifier. Then, all unsettled agents in the group with the largest group identifier in $G^{\prime}(u)$ can perform DFS and visit all nodes in $G\left(u^{\prime}\right)$ twice within $T$ steps. During the period, they pick up all stopped and unsettled agents in the component and goes back to the node that they are located at in the beginning of stage 2 .

Hence, each iteration of stages 1 and 2 decreases the number of nodes with at least one unsettled agent at least by half. This yields that all agents become settled and the dispersion is achieved within $O(T \log \ell)=O\left(\min \left(M, K \Delta, K^{2}\right) \log \ell\right)$ steps.

The knowledge of the global knowledge $M, K$, and $\Delta$ is inherent to the key idea of this algorithm. Without those upper bounds, the agents may switch between two stages before all agents complete a stage so that the correctness is no longer guaranteed or the agents may stay in one stage too long so that the running time is much larger than, for example, $\Omega(m n)$. To the best of our knowledge, no simple modification removes the requirement of the knowledge keeping the same running time asymptotically.

\section{Proposed Algorithm}

In this section, we give an algorithm $\mathcal{A}_{s v l}$ that solves a dispersion within $O\left(m^{\prime} \log \ell\right)$ steps and uses $O\left(\log \left(k+\delta_{\max }\right)\right)$ bits of memory space per agent. Algorithm $\mathcal{A}_{s v l}$ requires no global knowledge.

\subsection{Overview}

Algorithm $\mathcal{A}_{s v l}$ is based on the zombie algorithm explained in Section 3.3 but it has more sophisticated mechanism to achieve dispersion within $O\left(m^{\prime} \log \ell\right)$ steps. An agent $a$ maintains its mode on a variable $a$ mode $\in\{L, Z, S\}$. We say that an agent $a$ is a leader (resp. a zombie, a settled agent) when $a$.mode $=L$ (resp. $a$ mode $=Z, a$ mode $=S$ ). A leader may become a zombie, and a zombie eventually becomes a settled agent. However, a zombie never becomes a leader again, and a settled agent never changes its mode. An agent $a$ also maintains its level on a variable $a .1 \mathrm{v}$, while $a$ stores the identifier of some leader on a variable $a$.leader. As long as an agent $l$ is a leader, $l . \mathrm{ID}=l$.leader holds. These two variables, $1 \mathrm{v}$ and leader, determine the strength of agent $a$. We say that an agent $a$ is stronger than an agent $b$ if $a .1 \mathrm{v}>b .1 \mathrm{v}$ or $a .1 \mathrm{v}=b .1 \mathrm{v} \wedge a$. leader $>b$. leader holds.

All agents are leaders at the beginning of an execution of $\mathcal{A}_{s v l}$, Each time two or more leaders visit the same node, the strongest leader kills all other leaders, and the killed leaders become zombies. Zombies always follow the strongest leader that it has ever observed. A leader always tries to perform a DFS. Each time a leader visits an unsettled node $v$, the leader picks an arbitrary one zombie $z \in R(v)$ and makes $z$ settled. If there is no zombie at $v$, the leader suspends its DFS until zombies visits $v$. As in Section 3 we denote the settled node located at $v$ by $a_{v}$ if it exists. We say that a settled agent $s$ is a minion of a leader $l$ when $s .1 \mathrm{v}=l .1 \mathrm{v}$ and $s . l$ eader $=l$.ID hold. As in the zombie algorithm, a leader $l$ performs a DFS by using the memory space of its minions, in particular, by using the values of their lasts. When a leader $l$ visits a settled node $v$ such that $a_{v}$ is weaker than $l, a_{v}$ becomes a minion of $l$ by executing 
Table 2: Variables of $\mathcal{A}_{s v l}$

\begin{tabular}{|c|c|c|}
\hline variables & description & initial value \\
\hline$a . \operatorname{mode} \in\{L, Z, S\}$ & : $\quad$ the mode of an agent $a$ & $L$ \\
\hline a.slot $\in\{0,1,2,3\}$ & : the current timeslot & 0 \\
\hline$a .1 \mathrm{v} \in \mathbb{N}$ & the level of an agent $a$ & 0 \\
\hline a.leader $\in \mathbb{N}$ & the identifier of the strongest leader that $a$ observed & a.ID \\
\hline$a .1$ ast $\in \mathbb{N}$ & : the pointer to the strongest leader that $a$ has observed & 0 \\
\hline a.port $\in \mathbb{N} \cup\{-1\}$ & : the last non-negative value of pin & -1 \\
\hline
\end{tabular}

$\left(a_{v} .1 \mathrm{v}, a_{v}\right.$. leader $) \leftarrow(l .1 \mathrm{v}, l . \mathrm{ID})$. Conversely, the leader $l$ becomes a zombie if $a_{v}$ is stronger than $l$. At this time, the stronger leader may not be located at node $v$. However, $a_{v}$ can chase the stronger leader by moving via $a_{v}$.last repeatedly.

Each leader maintains its level like the famous minimum spanning tree algorithm given by Gallager, Humblet, and Spira 2. Initially, the level of every agent is zero. A leader increases its level by one each time it kills another leader with the same level or meets a zombie with the same level. We have the following lemma.

Lemma 1. No agent reaches a level larger than $\log _{2} \ell+1$.

Proof. Let $\ell_{2}$ be the nodes at which two or more leaders are located in the initial configuration, i.e., $\ell_{2}=|\{v \in V|| R(v, 0) \mid \geq 2\}|$. In step 0, (i) $\ell_{2}$ leaders changes their levels from 0 to 1 , (ii) the levels of $\ell-\ell_{2}$ leaders remain 0 , and (iii) other $k-\ell$ leaders become zombies with level 0 . Thus, at most $\ell_{2}+\left(\ell-\ell_{2}\right)=\ell$ leaders reaches level 1. Since a zombie never increases its level until it becomes a settled agent, for any $i \geq 1$, at most $\ell / 2^{i-1}$ leaders reaches level $i$. Thus, no agent reaches a level larger than $\log _{2} \ell+1$.

By definition of minions, a leader $l$ loses all its minions when $l$ increases its level by one. As we will see in Section 4.2, when a leader visits a node $v$, the leader considers that it has already visited a node $v$ in its DFS if and only if $a_{v}$ is a minion at that time. This means that a leader $l$ restarts a new DFS each time it increases its level. In every DFS, a leader $l$ moves at most $4 m^{\prime}$ times. However, this does not necessarily mean that $l$ completes its DFS or increases its level within $4 m^{\prime}$ steps because $l$ suspends its DFS while no other agent is located at the same node. Thus, we require a mechanism to bound the running time by $O\left(m^{\prime} \log \ell\right)$ steps. As we will see in Sections 4.2 and 4.3 , we achieve this by differentiating the moving speed of agents according to various conditions.

\subsection{Detailed Description}

The list of variables and the pseudocode of $\mathcal{A}_{s v l}$ are given in Table 2 and Algorithm 2 respectively.

First, we introduce some terminologies and notations. Let $t$ be any time step. We denote the set of leaders, the set of zombies, and the set of settled agents at step $t$ by $R_{L}(t), R_{Z}(t)$, and $R_{S}(t)$, respectively. In addition, we define $R_{L}(v, t)=R(v, t) \cap R_{L}(t), R_{Z}(v, t)=R(v, t) \cap R_{Z}(t)$, and $R_{S}(v, t)=R(v, t) \cap R_{S}(t)$ for any node $v \in V$. Define $R_{\max }(v, t)$ as the set of strongest agents in $R(v, t)$. We omit time step $t$ from those notation, e.g., simply write $R_{L}(v)$ for $R_{L}(v, t)$, when time step $t$ is clear from the context. A leader $l$ located at a node $v$ is called an active leader when $|R(v)| \geq 2$. When $|R(v)|=1, l$ is called a waiting leader. A zombie $z$ located at a node $v$ is called a strong zombie if $z .1 \mathrm{v}=a_{v} .1 \mathrm{v}$. Otherwise, $z$ is called a weak zombie. In the pseudocode, we use notation $a_{\max }$ for simplicity. We define $a_{\max }$ as follows: If there is a leader in $R_{\max }(v), a_{\max }$ denotes the (unique) leader in $R_{\max }(v)$; Otherwise, let $a_{\max }=a_{v}$.

${ }^{2}$ This definition assumes that there is at most one leader in $R_{\max }(v)$ and there is no zombie in $R_{\max }(v)$ at any time step. The former proposition holds because for any two leaders $l_{1}$ and $l_{2}, l_{1}$ is stronger than $l_{2}$, or $l_{2}$ is stronger than $l_{1}$. The latter holds because (i) a leader becomes a zombie only if it observes a stronger agent, (ii) a zombie never becomes stronger, i.e., never change its lv or leader, unless it becomes settled, and (iii) a zombie moves by chasing a stronger leader or moves together with a stronger leader. 


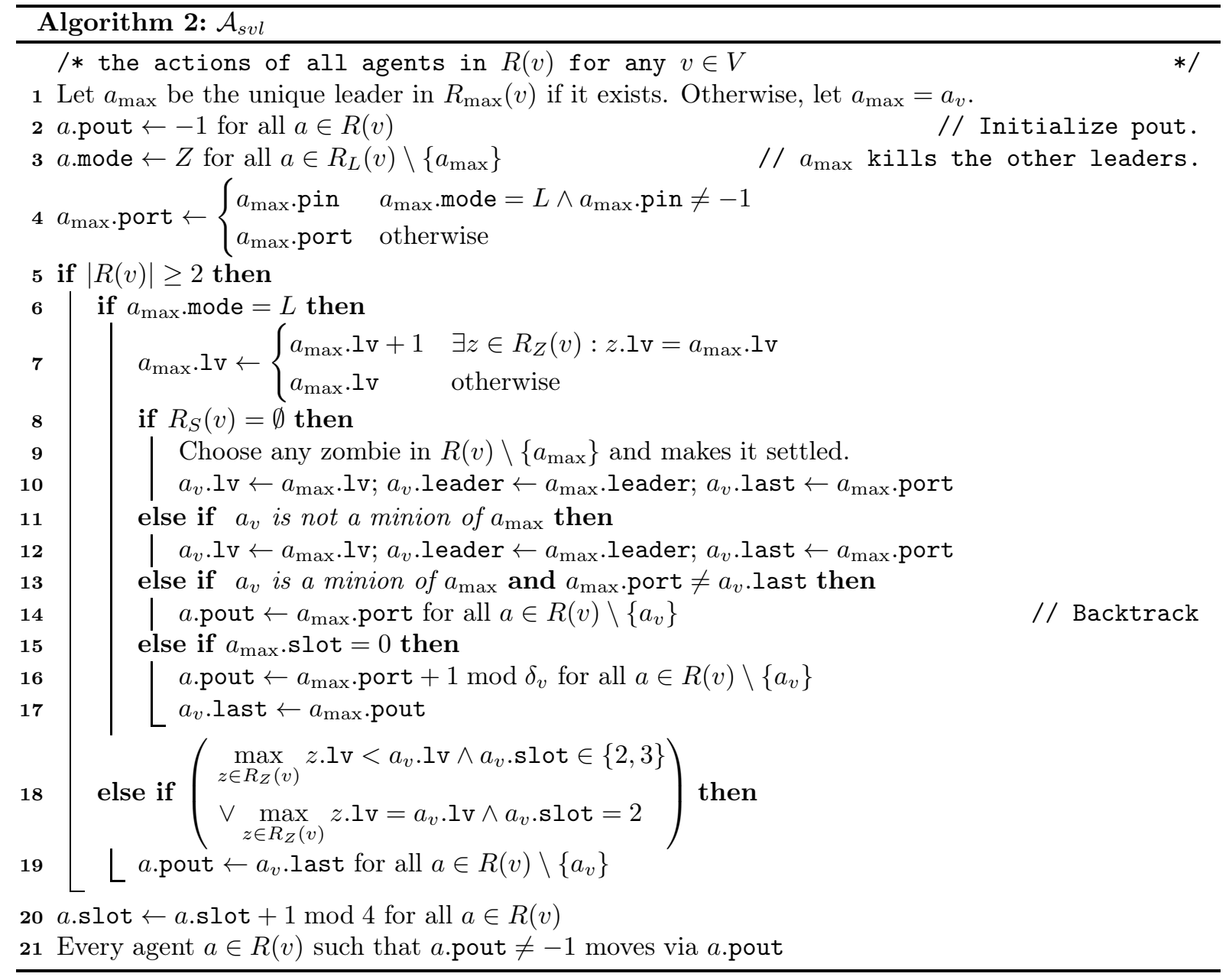


As mentioned in Section 4.1 we differentiate the moving speed of agents. We implement the differentiation with a variable slot $\in\{0,1,2,3\}$. Each agent $a$ counts how many steps have passed since an execution of $\mathcal{A}_{s v l}$ began modulo 4 and stores it on a.slot (Line 20). Thus, all agents always have the same value for this variable. This variable represents timeslots indicating which kind of agents are allowed to move at each step. For example, an active leader is allowed to move at slots 0 and 1, while a strong zombie is allowed to move only at slot 2 .

The pseudocode (Algorithm 2) specifies how the agents located at a node $v$ updates their variables including port pout, via which they move to the next destination. A leader is immediately killed, i.e., becomes a zombie, if it meets a stronger leader or a stronger settled agent (Line 3). A surviving leader increases its level by one each time it meets a (weaker) leader or zombie with the same level (Line 7). A leader $l$ performs a DFS using a variable last in its minions. It basically moves in timeslot 0 , while backtracking occurs in timeslot 1 . Since the information of incoming port in $l$.pin gets lost in timeslots 2 and 3, the leader $l$ remembers pin $=p_{v}(u)$ in a variable $l$.port each time $l$ moves from $u$ to $v$ (Line 4). Specifically, a leader $l$ performs a DFS as follows until it is killed by another leader:

- When $l$ moves from $u$ to $v$ such that $a_{v}$ is not a minion of $l$, the leader $l$ considers that it visits $v$ for the first time in the current DFS. Then, $l$ changes $a_{v}$ to a minion of $l$ (Line 12) and waits for the next timeslot 0 .

- When $l$ moves from $u$ to $v$ such that $v$ is an unsettled node, $l$ considers that it visits $v$ for the first time in the current DFS. If there is at least one zombie at $v, l$ changes arbitrary one zombie to a settled agent and make it a minion of $l$ (Lines 9 and 10). Otherwise, it suspends the current DFS until a zombie or a (weaker) leader $a$ visits $v$ (the condition in Line 5 implements this suspension). Then, $l$ does the same thing for $a$, i.e., make $a$ settled and a minion of $l$. Thereafter, $l$ waits for timeslot 0 .

- When $l$ moves from $u$ to $v$ such that $a_{v}$ is a minion and $a_{v}$.last $=l$.port, the leader $l$ considers that it has just backtracked from $u$ to $v$. Then, it just waits for the next time slot 0 .

- In timeslot $0, l$ leaves the current node $v$ via port $l$.port $+1=p_{v}(u)+1$ after storing this port on $a_{v}$.last (Lines 16 and 17).

- When $l$ moves from $u$ to $v$ such that $a_{v}$ is a minion and $a_{v}$.last $\neq l$.port, the leader $l$ considers that it has already visited $v$ before in the current DFS. Thus, it immediately backtracks from $v$ to $u$ without updating $a_{v}$.last (Line 15). Since a leader makes a non-backtracking move only in timeslot 0 , this backtracking occurs only in timeslot 1 .

In the first and second case, the leader $l$ simultaneously substitute $l$.port for $a_{v}$.last to avoid triggering backtracking mistakenly. Thus, each leader can perform a DFS in the same way as a simple DFS explained in Section 3.1 until it is killed and becomes a zombie.

A zombie always tries to follow a leader. If a zombie $z$ is located at a node $v$ without a leader, it moves via port $a_{v}$.last in timeslot 2 if $z$ is a strong zombie or some strong strong zombie is located at the same node $v$ (Line 19). Otherwise, $z$ moves via port $a_{v}$.last in timeslots 2 and 3 (Line 19). If $z$ is located at a node with a leader, it just moves with the leader (Lines 14 and 16) or becomes settled (Line $9)$.

\subsection{Correctness and Complexities}

In this section, we prove that an execution $\Xi_{\mathcal{A}_{s v l}}(C)$ of $\mathcal{A}_{s v l}$ starting from any configuration $C$ where all agents are in the initial state achieves dispersion within $O\left(m^{\prime} \log \ell\right)$ steps, and each agent uses $O(\log (k+$ $\left.\left.\delta_{\max }\right)\right)$ bits of memory space.

First, we gave an upper bound on the space complexity.

Lemma 2. Each agent uses at most $O\left(\log \left(k+\delta_{\max }\right)\right)$ bits of its memory space in an execution of $\mathcal{A}_{\text {svl }}$. 
Proof. Each agent $a$ maintains six non-constant variables: $a .1 \mathrm{v}, a$. leader, $a$. last, a.port, a.pin, and a.pout. The first variable $a .1 \mathrm{v}$ uses only $O(\log \log \ell)$ bits by Lemma 1 . Each of the other five variables just stores the identifier of some agent or the port numbers of some node. Thus, they uses only $O\left(\log \left(k+\delta_{\max }\right)\right)$ bits.

In an execution of $\mathcal{A}_{s v l}$, agents located at a node $v$ leaves $v$ only when there are two or more agents at $v$. Therefore, we have the following lemma.

Lemma 3. In an execution of $\mathcal{A}_{\text {svl }}$, once all agents are located at different nodes, no agent leaves the current location thereafter.

Thus, it suffices to show that all agents will be located at different nodes within $O\left(m^{\prime} \log n\right)$ steps in an execution of $\mathcal{A}_{s v l}$. To prove this, we introduce some terminologies and notations. Let $t$ be any time step and let $a$ be any agent in $R(v, t)$ for any $v$. Then, define the virtual level of agent $a$ at step $t$ as $V L(a, t)=\max _{b \in R(v)} b .1 \mathrm{v}$. Define $\mathcal{L}_{\text {min }}(t)$ be the minimum virtual level of all active leaders and all zombies in $R$ at step $t$. For simplicity, we define $\mathcal{L}_{\min }(t)=\infty$ if there is no active leader and no zombie in $R$ at step $t$. Again, we simply write $V L(a)$ and $\mathcal{L}_{\text {min }}$ for $V L(a, t)$ and $\mathcal{L}_{\text {min }}(t)$, respectively, when time step $t$ is clear from the context. Note that for any agent $a$, the virtual level and the level of $a$ differ if and only if $a$ is a weak zombie.

By definition of $\mathcal{A}_{s v l}$, we have the following lemma.

Lemma 4. In an execution of $\mathcal{A}_{\text {svl }}$, for any agent a, the virtual level of a never decreases.

Lemma 5. In an execution of $\mathcal{A}_{\text {svl }}$, from any time step, each active leader $l$ increases its level at least by one or becomes a waiting leader, a zombie, or a settled agent within $O\left(m^{\prime}\right)$ steps.

Proof. The active leader $l$ performs a DFS correctly unless it meets a leader/zombie with the same level or finds a stronger agent than $l$. Thus, it becomes a waiting leader if such event does not occur for sufficiently large $O\left(\mathrm{~m}^{\prime}\right)$ steps, Otherwise, it increases its level by one or becomes a zombie or a settled agent within $O\left(m^{\prime}\right)$ steps.

Lemma 6. In an execution of $\mathcal{A}_{\text {svl }}$, from any time step, each weak zombie $z$ increases its virtual level or catches up a leader or a strong zombie within $O(k)$ steps.

Proof. Suppose that now $z$ is located at a node $v$ and has a virtual level $i$. By definition of $\mathcal{A}_{s v l}$, we must have a path $w=v_{1}, v_{2}, \ldots, v_{s}$ such that $s \leq k, v=v_{1}, p_{v_{i}}\left(v_{i+1}\right)=a_{v_{i}}$. last for $i=1,2, \ldots, s-1$, and a leader with at least level $i$ is located at $v_{s}$. Let $j$ be the smallest integer such that a leader, a strong zombie, or an agent with virtual level $i^{\prime} \geq i+1$ is located at $v_{j}$. Then, sub-path $w^{\prime}$ of $w$ is defined as $w^{\prime}=v_{1}, v_{2}, \ldots, v_{j}$. This sub-path $w^{\prime}$ changes as time passes. Zombie $z$ moves forward in $w^{\prime}$ two times in every four steps because it moves in timeslots 2 and 3. However, a leader moves only once or moves and backtracks in every four steps, while a strong zombie moves only once in every four steps. Therefore, by Lemma 4 , the length of the $w^{\prime}$ decreases at least by one in every four steps, from which the lemma follows.

Lemma 7. In an execution of $\Xi=\Xi_{\mathcal{A}_{\text {svl }}}\left(C_{0}\right)=C_{0}, C_{1}, \ldots$, from any time step such that $\mathcal{L}_{\text {min }}<\infty$, $\mathcal{L}_{\text {min }}$ increases at least by one in $O\left(m^{\prime}\right)$ steps.

Proof. Let $C_{t}$ be any configuration where $\mathcal{L}_{\text {min }}=i$ holds. It suffices to show that the suffix $\Xi^{\prime}$ of $\Xi$ after $C_{t}$, i.e., $\Xi^{\prime}=C_{t}, C_{t+1}, \ldots$ reaches a configuration where $\mathcal{L}_{\min } \geq i+1$ holds within $O\left(m^{\prime}\right)$ steps. Let $R_{\gamma}$ be the set of all weak zombies with virtual level $i$ that are not located at a node with a leader or a strong zombie. No agent in $R \backslash R_{\gamma}$ becomes an agent in $R_{\gamma}$ in $\Xi^{\prime}$ because all leaders and all strong zombies must have levels no less than $i$, and thus they become weak zombies only after their virtual levels reach $i+1$. Therefore, by Lemma 6. $R_{\gamma}=\emptyset$ holds within $O\left(k^{\prime}\right)$ steps in $\Xi^{\prime}$, and $R_{\gamma}=\emptyset$ always hold thereafter. If there is no agent in $R_{\gamma}$, no waiting leader with at most level $i$ goes back to an active leader without increasing its level. Thus, by Lemma 5 , within $O\left(m^{\prime}\right)$ steps, $\Xi$ reaches a configuration $C_{t^{\prime}}$ from which there is always no active leader with level $i$. 
Let $\Xi^{\prime \prime}$ be the suffix $C_{t^{\prime}}, C_{t^{\prime}+1}, \ldots$ of $\Xi^{\prime}$. By definition, in $\Xi^{\prime \prime}$, there is no active leader with level at most $i$. In addition, the virtual level of a zombie is $i$ only if it is located at a node with a strong zombie with level $i$. Thus, it suffices to show that every strong zombie $z$ with level $i$ increases its virtual level at least by one within $O\left(\mathrm{~m}^{\prime}\right)$ steps. Suppose that $z$ is located at a node $v$. By definition of $\mathcal{A}_{s v l}$, we must have a path $w=v_{1}, v_{2}, \ldots, v_{s}$ such that $s \leq k, v=v_{1}, p_{v_{i}}\left(v_{i+1}\right)=a_{v_{i}}$. last for $i=1,2, \ldots, s-1$, and a leader $l$ is located at $v_{s}$. In $\Xi^{\prime \prime}$, there is no active leader with level $i$. Thus, if $l$ is active, its level is at least $i+1$. Even if $l$ is waiting, its level is at least $i$, and the virtual level of $a_{v_{s}}$ immediately becomes at least $i+1$ after an active leader or a zombie visits $v_{s}$. Therefore, if none of the agents $a_{v_{1}}, a_{v_{2}}, \ldots, a_{v_{s-1}}$ changes the value of its last during the next $4 s \leq 4 k$ steps, the virtual level of $z$ reaches at least $i+1$. Thus, suppose that some $a_{v_{j}}(1 \leq j \leq s-1)$ changes the value of its last during the $4 s$ steps. This yields that an active leader must visit $v_{j}$ during the period. Moreover, in $\Xi^{\prime \prime}$, every active leader has level at least $i+1$. Thus, the virtual level of agent $a_{v_{j}}$ must reach at least $i+1$ at that time. Therefore, in any case, zombie $z$ increases its virtual level at least by one within $O(k)$ steps in $\Xi^{\prime \prime}$.

Theorem 1. Algorithm $\mathcal{A}_{\text {svl }}$ solves a dispersion problem within $O\left(m^{\prime} \log \ell\right)$ steps. It uses $O\left(\log \left(k+\delta_{\max }\right)\right)$ bits of memory space per agent.

Proof. By Lemmas 1 and 17, $\mathcal{L}_{\text {min }}=\infty$ holds within $O\left(m^{\prime} \log \ell\right)$ steps. Since $\mathcal{L}_{\text {min }}=\infty$ yields that no active leader and no zombie exists, thus all agents are located at different nodes at that time. Therefore, the theorem immediately follows from Lemmas 2 and 3 .

\section{Conclusion}

In this paper, we presented a both time and space efficient algorithm for the dispersion problem. This algorithm does not require any global knowledge. However, we require that all agents compute and move synchronously. The proposed algorithm inherently requires the synchronous assumption: active leaders, strong zombies, and weak zombies move in different speeds. We leave open whether both time and space efficient algorithm can be designed for the asynchronous setting.

\section{References}

[1] John Augustine and William K. Moses. Dispersion of mobile robots. Proceedings of the 19th International Conference on Distributed Computing and Networking, Jan 2018.

[2] Robert G. Gallager, Pierre A. Humblet, and Philip M. Spira. A distributed algorithm for minimumweight spanning trees. ACM Transactions on Programming Languages and systems (TOPLAS), $5(1): 66-77,1983$.

[3] Ajay D Kshemkalyani and Faizan Ali. Efficient dispersion of mobile robots on graphs. In Proceedings of the 20th International Conference on Distributed Computing and Networking, pages 218-227, 2019.

[4] Ajay D Kshemkalyani, Anisur Rahaman Molla, and Gokarna Sharma. Efficient dispersion of mobile robots on arbitrary graphs and grids. arXiv preprint arXiv:1812.05352, 2018.

[5] Ajay D Kshemkalyani, Anisur Rahaman Molla, and Gokarna Sharma. Dispersion of mobile robots in the global communication model. In Proceedings of the 21st International Conference on Distributed Computing and Networking, pages 1-10, 2020.

[6] Petrişor Panaite and Andrzej Pelc. Exploring unknown undirected graphs. Journal of Algorithms, 33(2):281-295, 1999.

[7] Vyatcheslav B Priezzhev, Deepak Dhar, Abhishek Dhar, and Supriya Krishnamurthy. Eulerian walkers as a model of self-organized criticality. Physical Review Letters, 77(25):5079, 1996. 
[8] Yuichi Sudo, Daisuke Baba, Junya Nakamura, Fukuhito Ooshita, Hirotsugu Kakugawa, and Toshimitsu Masuzawa. A single agent exploration in unknown undirected graphs with whiteboards. IEICE Transactions on Fundamentals of Electronics, Communications and Computer Sciences, 98(10):2117$2128,2015$.

[9] Vladimir Yanovski, Israel A Wagner, and Alfred M Bruckstein. A distributed ant algorithm for efficiently patrolling a network. Algorithmica, 37(3):165-186, 2003. 